\title{
Identificación Hematológica y Molecular de Anaplasma platys en Caninos Domésticos de Lima Metropolitana con Signos Clínicos Compatibles con Anaplasmosis
}

\author{
Hematologic and Molecular Identification of Anaplasma platys in Domestic \\ Canine in Lima with Clinic Signs Compatible with Anaplasmosis
}

\author{
Viviana Tateishi T. ${ }^{1}$, Olga Lí E. ${ }^{13}$, Luis Hoyos S. ${ }^{1}$, Hermelinda Rivera G. ${ }^{2}$, \\ Alberto Manchego S. ${ }^{2}$, Luis Barrios A. ${ }^{1}$, Juan More B. ${ }^{2}$
}

\section{Resumen}

El presente estudio tuvo como objetivo determinar la presencia de Anaplasma platys en caninos domésticos de Lima Metropolitana con signos clínicos compatibles con anaplasmosis, mediante la identificación de corpúsculos de inclusión en plaquetas y a través de la técnica Hemi-Nested PCR en muestras de sangre periférica. Se recolectaron 144 muestras de sangre entre enero y diciembre de 2012. De estas, el 29.2\% (42/144) fue positiva (individuos trombocitopénicos con presencia de corpúsculos de inclusión en plaquetas) y el 12.5\% (18/144) resultó sospechoso (individuos no trombocitopénicos y presencia de corpúsculos de inclusión en plaquetas) a la identificación hematológica. Asimismo, 1.4\% (2/144) resultó positivo a la prueba de Hemi-Nested PCR. Estos hallazgos confirman la presencia de A. platys en caninos domésticos en el país.

Palabras clave: Anaplasma platys; hematología; corpúsculos de inclusión; plaquetas; Hemi-Nested PCR

\section{Abstract}

This study aimed to determine the presence of Anaplasma platys in domestic dogs of Metropolitan Lima with clinic signs consistent with anaplasmosis by identifying inclusion bodies in platelets and through the Hemi-Nested PCR technique using peripheral blood samples. For this purpose, 144 samples were collected between January to December 2012. The results showed that $29.2 \%$ (42/144) were positive (thrombocytopenic individuals

\footnotetext{
${ }^{1}$ Laboratorio de Patología Clínica y Biología Molecular, ${ }^{2}$ Laboratorio de Microbiología y Parasitología Veterinaria, Facultad de Medicina Veterinaria, Universidad Nacional Mayor de San Marcos, Lima, Perú

${ }^{3}$ E-mail: olgalie@hotmail.com
}

Recibido: 24 de julio de 2014

Aceptado para publicación: 16 de noviembre de 2014 
and presence of inclusion bodies in platelets) and $12.5 \%$ (18/144) were suspicious (nonthrombocytopenic individuals and presence of inclusion bodies in platelets) by hematologic identification. Moreover, 1.4\% (2/144) resulted positive to the Hemi-Nested PCR test. These results confirm the presence of A. platys in domestic dogs in the country.

Key words: Anaplasma platys; hematology; inclusion bodies; platelets; Hemi-Nested PCR

\section{INTRODUCCIÓN}

La trombocitopenia cíclica infecciosa canina o anaplasmosis trombocítica es una enfermedad de tipo infecciosa causada por Anaplasma platys, descrita por primera vez a fines de la década del 70 por Harvey et al. (1978). Posteriormente fue reclasificada como parte del género Anaplasma, dentro de la familia Anaplasmataceae (Dumler et al., 2001). Esta bacteria intracelular obligada, gram negativa, presenta una afinidad hacia las plaquetas de los caninos (Arraga-Alvarado et al., 2003) y se transmite principalmente por la picadura de garrapatas (Greene, 1997) del tipo Rhipicephalus sanguineus (Sainz et al., 1999; Inokuma et al., 2000).

A. platys ocasiona cuadros de trombocitopenia cíclica que pueden durar entre 7 y 14 días (Ettinger, 1992). Esta trombocitopenia es aparentemente de tipo regenerativa, debido a la hiperplasia megacariocítica encontrada en la médula ósea en perros infectados experimentalmente (Gaunt et al., 1990). Se pueden observar signos clínicos inespecíficos leves como pirexia y anorexia, así como petequias y equimosis (Greene, 1997), que tienden a agravarse en casos de co-infección con Ehrlichia canis (agente causal de la ehrlichiosis monocítica canina).
Existen reportes de A. platys en varios países del continente americano. Oscherov

et al. (2011) detectaron en garrapatas Rhipicephalus sanguineus la presencia de ADN de A. phagocitophylum (agente causal de la anaplasmosis granulocítica humana) y A. platys en el noreste de Argentina, mientras que en Brasil se ha detectado la enfermedad en caninos al realizar estudios comparativos entre técnicas hematológicas y moleculares (Ferreira et al., 2007), así como coinfecciones entre A. platys y Ehrlichia canis mediante PCR (Da Silva et al., 2012).

En Chile se ha detectado la presencia de A. platys mediante PCR (Abarca et al., 2007), además de anticuerpos contra Anaplasma en personas que convivían con caninos infectados (Abarca et al., 2008). Por otro lado, en Venezuela se detectó A. platys en trombocitos de caninos (Arraga-Alvarado et al., 2003; Huang et al., 2005) y en humanos con VIH (Tamí y Tamí-Maury, 2004).

Las investigaciones sobre enfermedades transmitidas por vectores son de gran importancia en el país, sobre todo por la amplia distribución de microorganismos con potencial zoonótico y, más aún, de aquellos vectores biológicos que contribuyen en su propagación. Por esta razón, se llevó a cabo el presente estudio para caracterizar hematológica y molecularmente a $A$. platys en sangre periférica de caninos domésticos provenientes de Lima Metropolitana. 


\section{Materiales y MéTodos}

\section{Selección de Canes}

Entre enero y diciembre de 2012 se recolectaron 144 muestras de sangre periférica venosa de caninos domésticos, pacientes de la Clínica de Animales Menores (CAM) de la Facultad de Medicina Veterinaria (FMV), Universidad Nacional Mayor de San Marcos (UNMSM), ubicada en el distrito de San Borja, Lima, Perú. Los animales fueron seleccionados según la presencia de signos clínicos compatibles con trombocitopenia cíclica infecciosa canina, e indistintamente del sexo y edad. El tamaño muestral establecido fue de 144 canes, de acuerdo a la fórmula para poblaciones infinitas (Daniel, 1996).

Los signos clínicos (criterios de inclusión) que se consideraron compatibles con anaplasmosis canina fueron depresión, anorexia, pirexia, palidez de mucosas, signos hemorrágicos (petequias, equimosis y uveítis anterior), linfadenomegalia, esplenomegalia y hepatomegalia. El estudio cumplió con las normas éticas para la investigación establecidas por el Comité de Ética y Bienestar Animal de la FMV-UNMSM.

\section{Muestras y Análisis de Laboratorio}

Las muestras de sangre $(3 \mathrm{ml})$ fueron tomadas con anticoagulante EDTA. El análisis hematológico y estudio del frotis sanguíneo se hizo en forma inmediata a la toma de muestra en el Laboratorio de Patología Clínica, y una alícuota se guardó en viales de $1.5 \mathrm{ml} \mathrm{a}-20^{\circ} \mathrm{C}$ en el Laboratorio de Virología, ambos de la FMV-UNMSM.

El análisis hematológico se realizó utilizando el equipo automatizado Abacus Junior Vet 5 (Diatron Group, Hungría). Asimismo, 48 muestras (1/3 del total) fueron seleccionadas de manera aleatoria y procesadas bajo los métodos clásicos del laboratorio clínico, con la finalidad de contrastar los resultados obtenidos a partir del equipo automatizado.
Así, se determinó el recuento de glóbulos rojos $\left(\mathrm{x} 10^{6} / \mu \mathrm{l}\right)$ y blancos $\left(\mathrm{x} 10^{3} / \mu \mathrm{l}\right)$ con la cámara de Neubauer, hemoglobina $(\mathrm{g} / \mathrm{dl})$ mediante el método de la cianometahemoglobina, hematocrito $(\%)$ mediante el método del microhematocrito, y el recuento diferencial (\%) y plaquetario $\left(\mathrm{x} 10^{3} / \mu \mathrm{l}\right)$ a partir del frotis sanguíneo coloreado con tinción Wright.

Los valores hematológicos referenciales para caninos fueron establecidos por el Laboratorio de Patología Clínica de la FMVUNMSM (Texto-Guía Patología Clínica Veterinaria, 2012). La identificación de corpúsculos de inclusión (CI) compatibles con $A$. platys fue realizada a partir de los frotis de sangre y bajo la visualización en microscopio con objetivo de inmersión (100X). Para esto, se identificaron aleatoriamente 20 campos (10 en monocapa y 10 en la cola del frotis), y se hizo la lectura en tres oportunidades.

Finalmente, a nivel hematológico se consideraron como negativos a la infección por A. platys a aquellos individuos que no presentaron trombocitopenia y corpúsculos de inclusión compatibles con A. platys en plaquetas, pudiendo presentar otras citopenias (anemia, leucopenia). Los individuos sin trombocitopenia pero con presencia de corpúsculos de inclusión en plaquetas fueron considerados como sospechosos, y aquellos individuos trombocitopénicos con presencia de corpúsculos de inclusión en plaquetas fueron considerados positivos.

Para el análisis molecular de las muestras obtenidas se usó la técnica de HemiNested PCR para la identificación de ADN de $A$. platys empleando tres cebadores con secuencia de nucleótidos determinada, según lo indicado por Chang y Pan (1996).

\section{Análisis de Datos}

Se emplearon cuadros de estadística descriptiva para presentar los resultados obtenidos, utilizando medidas de tendencia central y dispersión. 


\section{Resultados}

El Cuadro 1 muestra los signos clínicos encontrados en los canes muestreados. Los resultados del análisis hematológico se presentan en el Cuadro 2. A nivel de serie eritrocítica, los valores promedio de hematocrito $(24 \pm 7.2 \%)$, hemoglobina ( $8.1 \pm$ $2.44 \mathrm{~g} / \mathrm{dl}$ ) y recuento de glóbulos rojos (3.67 $\left.\pm 1.18 \times 10^{6} / \mu \mathrm{l}\right)$ se encontraron por debajo de los valores referenciales para la especie. Del mismo modo, a nivel de serie leucocítica, el valor promedio hallado para el recuento total leucocitario fue de $20.18 \pm 17.23 \times 10^{3} / \mu 1$, estando por encima del rango referencial. Los valores porcentuales circulantes para neutrófilos, linfocitos, eosinófilos y monocitos no evidenciaron cambios notorios. Finalmente, la serie trombocítica presentó valores promedio ligeramente disminuidos $(178 \pm 160$ $\left.\mathrm{x} 10^{3} / \mu \mathrm{l}\right)$.
Cuadro 1. Signos clínicos en 144 canes de Lima Metropolitana con sospecha de anaplasmosis (Lima, 2013)

\begin{tabular}{lr}
\hline Signos clínicos & $(\%)$ \\
\hline Anorexia & 50.0 \\
Fiebre & 12.5 \\
Depresión/Letargia & 33.3 \\
Linfoadenomegalia & 12.5 \\
Esplenomegalia & 29.2 \\
Palidez de mucosas & 66.7 \\
Secreción nasal & 8.3 \\
Hepatomegalia & 16.7 \\
Disnea & 8.3 \\
Petequias/Equimosis & 4.2 \\
Epistaxis & 8.3 \\
Vómitos/Diarreas & 20.8 \\
\hline
\end{tabular}

Cuadro 2. Examen hematológico de muestras de sangre periférica de caninos con signos clínicos de anaplasmosis canina en Lima Metropolitana (2013)

\begin{tabular}{lcccc}
\hline Variable & Promedio & D.E. & Rango & $\begin{array}{c}\text { Valores } \\
\text { normales }\end{array}$ \\
\hline Recuento de eritrocitos $\left(\mathrm{x} 10^{6} / \mu \mathrm{l}\right)$ & 3.67 & 1.18 & $0.9-6.7$ & $(5.5-7.5)$ \\
Hemoglobina $(\mathrm{g} / \mathrm{dl})$ & 8.1 & 2.44 & $2.3-14.6$ & $(12-18)$ \\
Hematocrito $(\%)$ & 24 & 7.2 & $6-42$ & $(37-55)$ \\
Recuento de leucocitos $\left(\mathrm{x} 10^{3} / \mu \mathrm{l}\right)$ & 20.18 & 17.23 & $0.6-77.2$ & $(8-13)$ \\
- Abastonados $(\%)$ & 0.9 & 2.81 & $0-24$ & $(0-3)$ \\
- Segmentados $(\%)$ & 76.15 & 15.52 & $26-96$ & $(65-75)$ \\
- Linfocitos $(\%)$ & 22.45 & 16.53 & $1-87$ & $(15-30)$ \\
- Monocitos $(\%)$ & 0.1 & 0.79 & $0-3$ & $(1-4)$ \\
- Eosinófilos $(\%)$ & 0.7 & 1.30 & $0-7$ & $(1-5)$ \\
- Basófilos $(\%)$ & 0 & 0 & 0 & $(0-0.5)$ \\
Recuento de plaquetas $\left(\mathrm{x} 10^{3} / \mu \mathrm{l}\right)$ & 178 & 160 & $80-720$ & $(200-400)$ \\
\hline
\end{tabular}

${ }^{1}$ Rangos referenciales establecidos por el Laboratorio de Patología Clínica de la FMV-UNMSM 
Cuadro 3. Resultados comparativos entre el análisis hematológico (frotis) y los resultados del Hemi-Nested PCR para la detección de Anaplasma platys (Lima, 2013)

\begin{tabular}{lcccc}
\hline \multirow{2}{*}{ Hemi-Nested PCR } & \multicolumn{3}{c}{ Análisis hematológico } & Total \\
\cline { 2 - 5 } & Positivo & Sospechoso & Negativo \\
\hline Positivo & 0 & 2 & 0 & 2 \\
Negativo & 42 & 16 & 84 & 142 \\
\hline Total & 42 & 18 & 84 & 144 \\
\hline
\end{tabular}

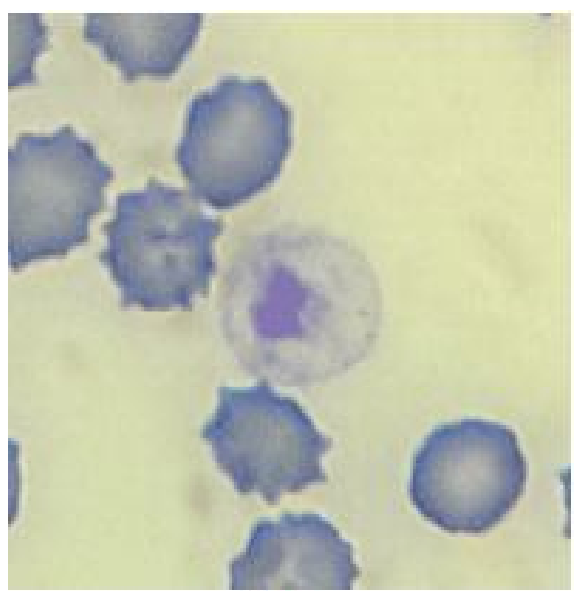

(a)

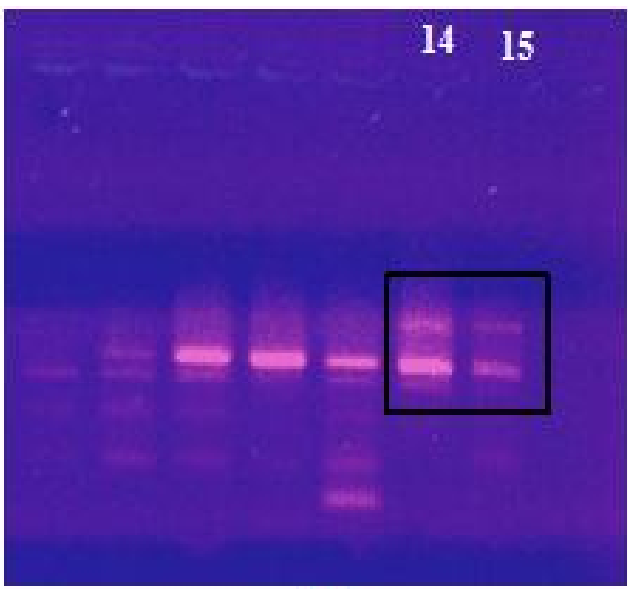

(b)

Figura 1. (a) Macroplaqueta con presencia de corpúsculo de inclusión basófilo en su citoplasma compatible con Anaplasma platys. Tinción Wright. 1000x. (b) Resultado positivo al ADN de A. platys en gel de agarosa al 1.5\% (pesos moleculares aproximados)

Las 48 muestras sanguíneas utilizadas para contrastar los resultados del equipo hematológico automatizado dieron resultados equivalentes en todos los parámetros hematológicos en estudio a las técnicas hematológicas de rutina utilizadas en el laboratorio.

A través del frotis sanguíneo se encontró $29.2 \%(42 / 144)$ de muestras positivas y $12.5 \%(18 / 144)$ de muestras sospechosas a anaplasmosis canina (Cuadro 2), en tanto que con la técnica de Hemi-Nested PCR (Fig. 1) solo se determinó la presencia del ADN de A. platys en $1.4 \%$ de los caninos (Cuadro 3 ).

\section{Discusión}

La evaluación hematológica reflejó, principalmente, $66.7 \%$ de muestras trombocitopénicas, frecuencia considerada como elevada. La presencia de un cuadro de trombocitopenia es común en el caso de infecciones por A. platys, aunque se desconoce con exactitud el mecanismo involucrado 
en la disminución del número de trombocitos (Eddlestone et al., 2007). Algunos autores plantean la posibilidad de que el microorganismo destruye las plaquetas (Harvey, 2012). Por otro lado, la escasa cantidad de plaquetas dificulta la posibilidad de detectar la bacteria (Chang y Pan, 1996; Ferreira et al., 2008).

La evaluación del frotis sanguíneo para determinar la presencia de corpúsculos de inclusión en plaquetas (Fig. 1a) evidenció 29.2 y $12.5 \%$ de muestras positivas y sospechosas. Pese a que el cuadro hematológico que se presenta en infecciones por A. platys es de característica cíclica (Harvey et al., 1978; Bradfield et al., 1996; Chang y Pan, 1996), es posible que estos resultados estén relacionados a la activación plaquetaria a causa de un proceso inflamatorio, probablemente generado por otro agente infeccioso que ocasiona estructuras similares a cuerpos de inclusión intraplaquetarios.

La ausencia de sintomatología hemorrágica en la mayoría de los perros incluidos en el estudio coincide con lo citado por Harvey et al. (1978) y Baker et al. (1987), quienes sugieren que las hemorragias no se presentan, posiblemente, por la poca duración de las trombocitopenias en esta enfermedad. Del mismo modo, se ha descrito que A. platys no llega a generar daños vasculares o vasculitis significativa en comparación con las infecciones por E. canis (Baker et al., 1987). En caso se manifieste, existen estudios que indican que puede estar relacionada a la disfunción plaquetaria producida por la rickettsia (Gaunt et al., 1990) o a coinfecciones con E. canis (Shaw, 2005).

El 1.4\% de muestras positivas A. platys mediante la técnica de Hemi-Nested PCR (Fig. 1b) se considera como un porcentaje bajo, comparado con otros estudios realizados en otros países donde la prevalencia fue mayor (Motoi et al., 2001; Huang et al., 2005; Martin et al., 2005; Da Silva et al., 2012). Finalmente, los resultados evidenciaron que pueden existir otras causas para la observación de corpúsculos de inclusión compatibles con A. platys frente a resultados negativos a PCR, tales como agregados proteínicos intracitoplasmáticos u otros agentes infecciosos (bacterianos y/o virales) (Ferreira et al., 2008).

\section{Conclusiones}

- El 29.2 y el $12.5 \%$ de 144 canes con sintomatología clínica compatible con anaplasmosis fue positiva y sospechosa a la enfermedad, respectivamente, de acuerdo a la presencia de trombocitopenia y corpúsculos de inclusión en plaquetas.

- El 1.4\% de los canes fue positivo al ADN de Anaplasma platys mediante la técnica de Hemi-Nested PCR.

\section{Literatura Citada}

1. Abarca K, López J, Perret C, Guerrero J, Godoy P, Veloz A, ValienteCheverría F, et al. 2007. Anaplasma platys in dogs, Chile. Emerg Infect Dis 13: 1392-1395. doi: 10.3201/ eid1309.070021

2. Abarca K, López J, González P, Dabanch J, Torres M, Solari V, Perret C. 2008. Evidencia seroepidemiológica de exposición humana a Anaplasma spp en Santiago, Chile. Rev Chil Infect 25: 358-361.

3. Arraga-Alvarado C, Palmar M, Parra O, Salas P. 2003. Ehrlichia platys (Anaplasma platys) in dogs from Maracaibo, Venezuela: an ultrastructural study of experimental and natural infections. Vet Pathol 40: 149-156. doi: 10.1354/vp.40-2-149

4. Baker DC, Simpsons M, Gaunt SD, Corstvet RE. 1987. Acute Ehrlichia platys infection in the dog. Vet Pathol 24:449-453. 
5. Bradfield JF, Vore SJ, Pryor WH. 1996. Ehrlichia platys infection on dogs. Lab Anim Sci 46: 565-568.

6. Chang WL, Pan MJ. 1996. Specific amplification of Ehrlichia platys DNA from blood specimens by two-step PCR. J Clin Microbiol 34: 3142-3146.

7. Da Silva GCF, Benitez AN, Girotto A, Taroda A, Vidotto MC, Garcia JL, et al. 2012. Occurrence of Ehrlichia canis and Anaplasma platys in household dogs from northern Parana. Rev Bras Parasitol Vet 21: 379-385. doi: 10.1590/S1984-29612012005000009

8. Daniel D. 1996. Bioestadística base para el análisis de las ciencias de la salud. $5^{\circ}$ ed. México: Limusa. 480 p.

9. Dumler JS, Barbet AF, Bekker CPJ, Dasch GA, Palmer GH, Ray SC, et al. 2001. Reorganization of genera in the families Rickettsiaceae and Anaplasmataceae in the order Rickettsiales: unification of some species of Ehrlichia with Anaplasma, Cowdria with Ehrlichia and Ehrlichia with Neorickettsia, descriptions of six new species combinations and designation of Ehrlichia equi and 'HGE agent' as subjective synonyms of Ehrlichia phagocytophila. Int J Syst Evol Microbiol 51: 2145-2165. doi: 10.1099/ 00207713-51-6-2145

10. Eddlestone SM, Gaunt SD, Neer TM, Boudreaux CM, Gill A, Haschke E, Corstvet RE. 2007. PCR detection of Anaplasma platys in blood and tissue of dogs during acute phase of experimental infection. Exp Parasitol 115: 205210. doi: 10.1016/j.exppara.2006.08.006

11. Ettinger SJ. 1992. Tratado de medicina interna. Enfermedades del perro y el gato. México: Intermédica. $2568 \mathrm{p}$.

12. Ferreira RF, Cerqueira AMF, Pereira AM, Guimarães CM, De Sá AG, Abreu FS, et al. 2007. Anaplasma platys diagnosis in dogs: comparison between morphological and molecular tests. Intern J Appl Res Vet Med 5: 113-119.
13. Ferreira RF, Cerqueira AMF, Ferreira MS, Almosny NRP. 2008. Hematologic parameters in polymerase chain reaction-positive and -negative dogs for Anaplasma platys presenting platelet inclusion bodies. Intern J Appl Res Vet Med 6: 185-190.

14. Gaunt SD, Baker DC, Babin SS. 1990. Platelet aggregation studies in dogs with acute Ehrlichia platys infection. Am J Vet Res 51: 290-293.

15. Greene RT. 1997. Ehrlichiosis canina: implicaciones clínicas de factores humorales. En: Kirk R (ed). Terapéutica veterinaria de pequeños animales. $12^{\circ}$ ed. México: McGraw-Hill Interamericana. p 317-320.

16. Harvey JW, Simpson CF, Gaskin JM. 1978. Cyclic thrombocytopenia induced by a Rickettsia-like agent in dogs. J Infect Dis 137: 182-188.

17. Harvey JW. 2012. Evaluation of hemostasis: coagulation and platelet disorders. In: Veterinary hematology: a diagnostic guide and color atlas. St. Louis, Missouri: Elsevier. p 209-215.

18. Huang H, Unver A, Perez MJ, Orellana NG, Rikihisa Y. 2005. Prevalence and molecular analysis of Anaplasma platys in dogs in Lara, Venezuela. Braz J Microbiol 36: 211-216. doi: 10.1590/S1517-83822005000300002

19. Inokuma HD, Raoult D, Brouqui P. 2000. Detection of Ehrlichia platys DNA in brown dog ticks (Rhipicephalus sanguineus) in Okinawa Island, Japan. J Clin Microbiol 38: 4219-4221.

20. Martin AR, Brown GK, Dunstan RH, Roberts TK. 2005. Anaplasma platys: an improved PCR for its detection in dogs. Exp Parasitol 109: 176-180. doi: 10.1016/j.exppara.2004.11.007

21. Motoi Y, Satoh H, Inokuma H, Kiyuuna T, Muramatsu Y, Ueno H, Morita C. 2001. First detection of Ehrlichia platys in dogs and ticks in Okinawa, Japan. Microbiol Immunol 45: 89-91. doi: 10.1111/j.1348-0421.2001. tb01263.x 
22. Oscherov EB, Milano AMF, Lobo B, Anda P, Escudero R. 2011. Detection of Anaplasma platys and other pathogens in ectoparasites from urban hosts in Northeast Argentine. Rev Ibero-Latinoam Parasitol 70(1): 42-48.

23. Sainz A, Amusategui I, Tesouro MA. 1999. Ehrlichia platys infection and disease in dogs in Spain. J Vet Diagn Invest 11: 382-384. doi: 10.1177/ 104063879901100419
24. Shaw S. 2005. Infectious canine cyclic thrombocytopenia: Anaplasma platys comb. Nov infection. In: Shaw SE, Day MJ (eds). Arthropod-borne infectious diseases of the dog and cat. London: Manson Publishing. p 133.

25. Tamí IC, Tami-Maury IM. 2004. Identificación morfológica de Ehrlichia sp en las plaquetas de pacientes con infección por virus de la inmunodeficiencia humana, en Venezuela. Rev Panam Salud Públ 16: 345-349. 